\title{
Replication Study and Meta-Analysis in European Samples Supports Association of the 3p21.1 Locus with Bipolar Disorder
}

Evangelos Vassos, Stacy Steinberg, Sven Cichon, Gerome Breen, Engilbert Sigurdsson, Ole A. Andreassen, Srdjan Djurovic, Gunnar Morken, Maria Grigoroiu-Serbanescu, Carmen C. Diaconu, Piotr M. Czerski, Joanna Hauser, Gulja Babadjanova, Lilia I. Abramova, Thomas W. Mühleisen, Markus M. Nöthen, Marcella Rietschel, Peter McGuffin, David St. Clair, Omar Gustafsson, Ingrid Melle, Olli P.H. Pietiläinen, Mirella Ruggeri, Sarah Tosato, Thomas Werge, Roel A. Ophoff, GROUP Consortium, Dan Rujescu, Anders D. Børglum, Ole Mors, Preben B. Mortensen, Ditte Demontis, Mads V. Hollegaard, Ruud van Winkel, Gunter Kenis, Marc De Hert, János M. Réthelyi, István Bitter, I. Alex Rubino, Vera Golimbet, Lambertus A. Kiemeney, Leonard H. van den Berg, Barbara Franke, Erik G. Jönsson, Anne Farmer, Hreinn Stefansson, Kari Stefansson, and David A. Collier

Background: Common genetic polymorphisms at chromosome 3p21.1, including rs2251219 in polybromo 1 (PBRM1), have been implicated in susceptibility to bipolar affective disorder (BP) through genome-wide association studies. Subsequent studies have suggested that this is also a risk locus for other psychiatric phenotypes, including major depression and schizophrenia.

Methods: To replicate the association, we studied 2562 cases with BP and 25,439 control subjects collected from seven cohorts with either genome-wide association or individual genotyping of rs2251219 and tagging single nucleotide polymorphisms across the PBRM1 gene. Results from the different case-control groups were combined with the inverse variance weighting method.

Results: In our dataset, rs2251219 was associated with BP (odds ratio [OR] $=.89, p=.003$ ), and meta-analysis of previously published data with our nonoverlapping new data confirmed genome-wide significant association ( $\mathrm{OR}=.875, p=2.68 \times 10^{-9}$ ). Genotypic data from the SGENE-plus consortium were used to examine the association of the same variant with schizophrenia in an overall sample of 8794 cases and 25,457 control subjects, but this was not statistically significant $(\mathrm{OR}=.97, p=.21)$.

Conclusions: There is strong evidence of association of rs2251219 with BP. However, our data do not support association of this marker with schizophrenia. Because the region of association has high linkage disequilibrium, forming a large haplotype block across many genes, it is not clear which gene is causally implicated in the disorder.

Key Words: 3p21.1, bipolar disorder, genetics, polybromo 1, genome-wide association, schizophrenia

From the MRC SGDP Centre (EV, GB, PM, AF, DAC), Institute of Psychiatry, King's College London, London; Institute of Medical Sciences (DSC), University of Aberdeen, Foresterhill, Aberdeen; Wellcome Trust (OPHP), Sanger Institute, Cambridge, United Kingdom; deCODE genetics (SS, OG, HS, KS); Department of Psychiatry (ES), National University Hospital; School of Medicine (ES), University of Iceland, Reykjavik, Iceland; Institute of Neuroscience and Medicine (INM-1) (SC), Research Center Juelich, Juelich; Institute of Human Genetics (SC, TWM, MMN); Department of Genomics, Life \& Brain Center (SC, TWM, MMN), University of Bonn; German Center for Neurodegenerative Disorders (DZNE) (MMN), Bonn, Germany; Department of Genetic Epidemiology in Psychiatry (MR), Central Institute of Mental Health Mannheim, University of Heidelberg, Mannheim; Division of Molecular and Clinical Neurobiology (DR), Department of Psychiatry, Ludwig-Maximilians University, Munich, Germany; Institute of Clinical Medicine (OAA, SD, IM), University of Oslo; Department of Medical Genetics (SD); Division of Mental Health and Addiction (OAA, OG, IM), Oslo University Hospital, Oslo; Department of Psychiatry (GM), St Olav's Hospital and Norwegian University of Science and Technology, Trondheim, Norway; Biometric Psychiatric Genetics Research Unit (MG-S), Alexandru Obregia Clinical Psychiatric Hospital; Stefan S. Nicolau Institute of Virology (CCD), Romanian Academy, Bucharest, Romania; Laboratory of Psychiatric Genetics (PMC, JH), Poznan University of Medical Sciences, Poznan, Poland; Institute of Pulmonology (GB) Russian State Medical University; National Research Center of Mental Health (LIA, VG), Russian Academy of Medical Science, Moscow, Russia; Institute of Molecular Medicine Finland FIMM (OPHP); Public Health Genomics Unit (OPHP), National Institute for Health and Welfare, Helsinki, Finland; Section of Psychiatry and Clinical Psychology (MR, ST), University of Verona, Verona; Department of Neuroscience (IAR), Section of Psychiatry, University of Rome - Tor Vergata, Rome, Italy; Research Institute of Biological Psychiatry (TW), Mental Health Centre Sct. Hans Copenhagen University Hospital, Roskilde; Center for Psychiatric Research (ADB, OM), Aarhus University Hospital, Risskov; Department of Human Genetics (ADB, DD), Department of Biomedicine; National Center for Register-Based Research (PBM), Aarhus University, Aarhus; Department of Clinical Biochemistry and Immunology (MVH), Statens Serum Institut, Copenhagen, Denmark; University of California at Los Angeles Center for Neurobehavioral Genetics (RAO), Los Angeles, California; Department of Psychiatry (RAO); Department of Neurology (LHvdB), Rudolf Magnus Institute of Neuroscience, University Medical Center Utrecht, Utrecht; Department of Psychiatry and Psychology (RVW), School of Mental Health and Neuroscience, European Graduate School of Neuroscience (EURON), South Limburg Mental Health Research and Teaching Network (SEARCH), Maastricht University Medical Centre, Maastricht; Department of Epidemiology and Biostatistics and Department of Urology (LAK); Departments of Human Genetics and Psychiatry (BF), Donders Institute for Brain, Cognition and Behavior, Radboud University Nijmegen Medical Centre, Nijmegen, The Netherlands; University Psychiatric Centre (RVW, GK, MDH), Catholic University Leuven, Kortenberg, Belgium; Department of Psychiatry and Psychotherapy (JMR, IB), Semmelweis University, Budapest, Hungary; and the Department of Clinical Neuroscience (EGJ), HUBIN Project, Karolinska Institutet and Hospital, Stockholm, Sweden. Address correspondence to Evangelos Vassos, M.D., King's College London, Institute of Psychiatry, MRC SGDP Centre, Box P082, De Crespigny Park, London, SE5 8AF, United Kingdom; E-mail: evangelos.vassos@iop.kcl.ac.uk.

Received Sep 16, 2011; revised Jan 23, 2012; accepted Feb 10, 2012. 
G enome-wide association studies (GWAS) have identified new loci associated with psychiatric diseases such as schizophrenia and mood disorders. The hypothesis-free strategy, the large samples involved in these studies, and the stringent criteria for genome-wide significance ensure higher confidence in the validity of these findings in comparison with the previous candidate gene approach. However, to increase our confidence in the findings and to estimate accurately the effect size of the associated variants, the confirmation of association signals through replication is still necessary (1).

Bipolar affective disorder (BP) was included among the seven original diseases studied with GWA by the Wellcome Trust Case Control Consortium (WTCCC) (2), and subsequent studies with larger samples have identified single nucleotide polymorphisms (SNPs) in CACNA1C, ANK3, DGKH and recently neurocan (NCAN) associated with BP $(3,4)$. A meta-analysis of GWAS (5) with 3683 cases and 14,507 control subjects identified three chromosomal regions (1p31.1, 3p21, and 5q15) potentially associated with BP. The association with 3p21.1 was confirmed by a subsequent metaanalysis (6). The authors suggested that this is a risk locus for mood disorders in general, providing support for the previous epidemiologic evidence of genetic overlap between BP and unipolar depression. However, further analysis of that and additional data failed to support a role for $3 p 21$ in unipolar depression (7). A recent study found evidence of association of the same genetic marker with schizophrenia and concluded that its effect crosses traditional diagnostic boundaries (8). A meta-analysis of all available genome-wide data by the Psychiatric Genomics Consortium (https://pgc. unc.edu/) identified a new intronic variant in ODZ4 and confirmed an association of SNPs at the 3p21.1 locus with a combined sample of BP and schizophrenia (9).

In the present study we tested the association of the most significant marker from the previous studies at this locus (rs2251219) with BP in a large collection of samples from seven European sites, and we performed a meta-analysis of our new data with the BP data from the study by McMahon et al. (6). Because the associated SNP lies in a genomic area of high linkage disequilibrium (LD) spanning 10 genes, we searched for potential functional polymorphisms in high LD with the index SNP rs2251219. Finally, we examined the association of this SNP with schizophrenia with data from the SGENE-plus consortium (10).

\section{Methods and Materials}

\section{Subjects}

All samples were of Caucasian ancestry and had a diagnosis of BP according to the guidelines of the ICD-9 or -10 (11) or the DSM-IV (12). All subjects provided written informed consent for participation in accordance with the local ethical committees requirements. The samples were recruited from seven different sites.

United Kingdom Sample. This consisted of two independent samples. One was from London (UK-BACC), comprising $402 \mathrm{pa-}$ tients interviewed with the Schedules for Clinical Assessment in Neuropsychiatry (13) and 467 control subjects interviewed face to face or by telephone with a modified version of the Past History Schedule (14). The second sample (UK-BIP) was composed of 359 cases who met DSM-IV criteria for bipolar I disorder, recruited through psychiatric hospitals in Scotland, and 145 control subjects. Consensus diagnosis and the Operational Criteria Checklist for Psychotic Illness program (15) were used to define diagnoses.

Icelandic Sample. The Icelandic sample consisted of 544 subjects with $B P$ and 23,750 population control subjects. Patients and control subjects were recruited from all over Iceland. For 303 sub- jects, diagnoses were assigned according to Research Diagnostic Criteria (16) through the use of the Schedule for Affective Disorders and Schizophrenia-Lifetime Version (17). For 82 subjects, DSM-IV BP diagnoses were obtained through the use of the Composite International Diagnostic Interview $(18,19)$. In addition, there were 150 subjects with ICD-9 or -10 BP diagnoses, and 9 subjects with DSM-III BP clinical diagnoses. The 23,750 control subjects were recruited as a part of various genetic programs at deCODE Genetics (Reykjavik, Iceland) and were not screened for psychiatric disorders.

Norwegian Sample. The Norwegian sample consisted of 423 cases and 398 control subjects. Cases were recruited from outpatient and inpatient psychiatric units, and diagnoses were established with the Structured Clinical Interview for DSM-IV-TR-Axis I Disorders (20). The healthy control subjects were randomly selected from the Oslo and Akershus region and were screened by interview for severe mental illness with the Primary Care Evaluation of Mental Disorders (21). They were excluded if they or any of their close relatives had a lifetime history of a severe psychiatric disorder (schizophrenia, BP, and major depression). Further exclusion criteria were history of moderate/severe head injury, neurological disorder, mental retardation, medical problems thought to interfere with brain function (hypothyroidism, uncontrolled hypertension, and diabetes), and significant illicit drug use (22).

Polish, Russian, Romanian Samples. All patients were recruited from consecutive hospital admissions and were directly interviewed with the Structured Clinical Interview for DSM-IV-TRAxis I Disorders Patient Version. Information provided by medical records and interviews of family members was also used in a best estimate procedure of diagnosis on the basis of DSM-IV-TR criteria. The control samples were population-based, drawn from the same population as the patients. Only the Romanian control sample was screened for major psychiatric disorders. The ethnicity of the patients and control subjects was determined by genealogical investigation to the grandparental generation (23). Numbers of cases and control subjects/sample are presented in Table 1.

\section{SNP Selection and Genotyping}

Genomic DNA was isolated from whole blood samples or buccal mucosa swabs with standard methods at the recruitment sites. The rs2251219 SNP (top hit in the region in the WTCCC GWA and McMahon et al. studies $[2,6])$ was genotyped in all samples. In a subset of this sample, we performed additional genotyping of 12 tagging SNPs (rs10510760, rs1108842, rs11715796, rs13086936, rs17477867, rs2071508, rs2230535, rs2336142, rs2710322, rs3755799, rs7549, rs9879090) identified with "tagger" (http://www.broadinstitute.org/ $\mathrm{mpg} /$ tagger/) across our candidate gene at the time, polybromo 1 (PBRM1), in an attempt to refine the associated locus. Pairwise tagging was used, with an $r^{2}$ threshold of .8. Other parameters were left at the default settings.

The UK samples were genotyped on an ABI $7900 \mathrm{HT}$ Real Time PCR system (Applied Biosystems, Foster City, California) with standard Taqman genotyping assays at the MRC, SGDP Centre, Institute of Psychiatry, London, United Kingdom. The Icelandic sample was genotyped with HumanHap300 and HumanCNV370 Bead Arrays (Illumina, San Diego, California) at deCODE Genetics (Reykjavik, Iceland), and the Norwegian sample was genotyped with the Affymetrix Gene Chip Genome-Wide SNP 6.0 array (Affymetrix, Santa Clara, California). The sample call rate was at least $98 \%$ for the Illumina arrays and at least $95 \%$ for the Affymetrix array. Genotyping of all the remaining national samples was performed with the MassARRAY system on a Sequenom Compact MALDI-TOF device (Sequenom, San Diego, California) at the Department of Genomics, Life and Brain Center of the University of Bonn, Germany. 
Table 1. Association of rs $2251219-C$ with BP in Our Sample

\begin{tabular}{lcccrc}
\hline Sample & $\begin{array}{c}\text { Frequency } \\
\text { (cases) }\end{array}$ & $\begin{array}{c}\text { Frequency } \\
\text { (control } \\
\text { subjects) }\end{array}$ & OR (95\% Cl) & $\begin{array}{c}n \\
\text { (cases) }\end{array}$ & $\begin{array}{c}n \text { (control } \\
\text { subjects) }\end{array}$ \\
\hline Iceland & .369 & .410 & $.84(.74-.96)$ & 543 & 23,549 \\
Norway & .402 & .404 & $.99(.81-1.21)$ & 419 & 396 \\
Poland & .421 & .459 & $.86(.71-1.03)$ & 404 & .010 \\
Romania & .461 & .421 & $1.18(.88-1.58)$ & 193 & .939 \\
Russia & .430 & .456 & $.90(.69-1.17)$ & 242 & .106 \\
UK-BACC & .345 & .398 & $.79(.65-.96)$ & 402 & .277 \\
UK-BIP & .401 & .403 & $.99(.75-1.31)$ & 359 & .434 \\
\hline
\end{tabular}

$\mathrm{BP}$, bipolar disorder; $\mathrm{Cl}$, confidence interval; $\mathrm{OR}$, odds ratio.

\section{Statistical Analysis}

Control subjects from all samples were tested for deviation from Hardy-Weinberg Equilibrium, and genotyping yield was at least $95 \%$ in cases and control subjects of all groups with the exception of the marker rs9879090 in the Polish sample (91\% in cases; $94 \%$ in control subjects). A likelihood procedure implemented in the NEMO software developed at deCODE Genetics was used for the association analyses (24). Allele-specific odds ratios (ORs) and associated $p$ values were calculated, assuming a multiplicative model for the two chromosomes of an individual. Genomic control correction was applied in the Icelandic and Norwegian samples that were genotyped with genome-wide arrays. Results from the different case-control groups were combined with the fixed effect (inverse variance) method. Evidence for heterogeneity between samples was assessed with Cochran's Q and I I $^{2}$ statistics (25).

Because the allele frequency in cases and control subjects was not available for the GSK-BP sample, to combine our data with those presented in the study by McMahon et al. (6), we calculated the standard error of the OR from the given $p$ values and 95\% confidence interval $(\mathrm{Cl})$ and used the inverse-variance weighting method in Stata software version 10 (26). To avoid duplicated samples in the meta-analysis with published data, we excluded both samples from the United Kingdom. The BACC sample was partly included in the WTCCC study (2) and partly in the GSK-BP sample (5). The UK-BIP sample was partly included in the WTCCC study, but because we could not identify with certainty the individuals already included, we decided to exclude the whole sample. For the WTCCC cohort included in the meta-analysis additional genotypic data for control subjects from the WTCCC2 (http://www.wtccc.org.uk/) were available, raising the total number of control subjects to 5461.

Finally, we performed a cross disorder analysis with schizophrenia with data from the SGENE-plus consortium to determine whether the genetic risk of BP conferred by the 3p21.1 locus is shared with schizophrenia. For this analysis the total sample included 8794 cases and 25,457 control subjects from 22 different sites. Genomic control correction was applied to all genome-wide typed samples, and results from the different sites were pooled together with the Mantel-Haenzel model. For the power calculations, the effective sample size for the complete study was computed as the sum of the effective sample size of each group. Detailed description of the sample recruitment and genotyping methods is presented in Steinberg et al. (27).

\section{Results}

Our combined sample showed association at the same direction as the study by McMahon et al. (6) with the T allele increasing the risk for BP (Table 1). Genotypes in all control groups were in HardyWeinberg equilibrium $(p>.01)$. The pooled OR in our sample (2562 cases with BP, and 25,439 control subjects) was .89 (95\% Cl: .82-.96, $p=.003$ ). All of the additional tagging SNPs we genotyped showed similar or less significant association (Table S1 in Supplement 1).

Our data from the SGENE-plus consortium with genotyping of 8794 cases with schizophrenia and 25,457 control subjects (Table S2 in Supplement 1) did not confirm the association of rs2251219 with schizophrenia (OR $=.97, p=.21$ ). The power of our sample to detect significant association $(\alpha=.05)$ with the effect size observed for BP in this study or that reported for schizophrenia by Williams et al. (8) estimated with the Genetic Power Calculator (28) was close to 1.

To combine all available data, we performed a meta-analysis of our new data with the data presented by McMahon et al. after excluding the United Kingdom samples (761 cases and 511 control subjects) due to partial overlap with samples included in the previous meta-analysis. We used the fixed effect model as there was not significant heterogeneity between the samples $(Q=9.8, Q p$ value $=.28, \mathrm{I}^{2}=18 \%$ ). The pooled OR in the total data is estimated at $.875,95 \% \mathrm{Cl}: .84-.91, p=2.68 \times 10^{-9}$. The forest plot of the meta-analysis is presented in Figure 1.

A proxy search for SNPs in high LD with our index SNP rs2251219 was performed on the SNAP website (http://www.broadinstitute. org/mpg/snap/ldsearch.php) with the European panel from the 1000 genomes project [29] pilot dataset. This identified 195 SNPs in high LD $\left(r^{2}>\right.$ 8) with rs2251219. Among these there were 4 nonsynonymous SNPs, 3 SNPs that occur at splice sites, and 2 SNPs in

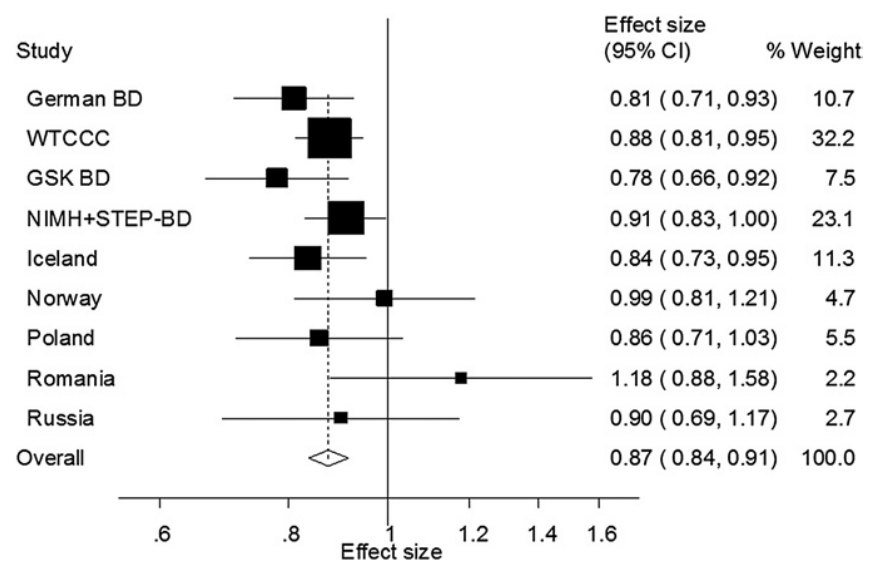

Figure 1. Forest plot of odds ratios with $95 \%$ confidence interval $(\mathrm{Cl})$ for the total bipolar disorder (BD) samples included in the meta-analysis. GSK, GlaxoSmithKline; NIMH, National Institute of Mental Health; STEP, Systematic Treatment for Enhancement Program; WTCCC, Wellcome Trust Case Control Consortium. 
Table 2. SNPs in the LD Area with Potentially Functional Role on Genes

\begin{tabular}{|c|c|c|c|c|c|c|c|}
\hline SNP & Position & Distance & $R^{2}$ & $\mathrm{D}^{\prime}$ & MAF & Function & $\begin{array}{l}\text { Gene } \\
\text { Name }\end{array}$ \\
\hline rs34005367 & 52533944 & 25883 & .811 & 1 & .417 & Splice site & NT5DC2 \\
\hline rs4234633 & 52532078 & 27749 & .811 & 1 & .417 & Splice site & STAB1 \\
\hline rs11719685 & 52651105 & 91278 & .965 & 1 & .375 & Splice site & PBRM1 \\
\hline rs11177 & 52696345 & 136518 & .965 & 1 & .375 & Nonsynonymous & GNL3 \\
\hline rs2289247 & 52702297 & 142470 & .899 & 1 & .392 & Nonsynonymous & GNL3 \\
\hline rs6976 & 52703844 & 144017 & .965 & 1 & .375 & Nonsynonymous & GLT8D1 \\
\hline rs8906 & 52714560 & 154733 & .965 & 1 & .375 & $5^{\prime}$ UTR & GLT8D1 \\
\hline rs6617 & 52715222 & 155395 & .899 & 1 & .392 & $5^{\prime}$ UTR & SPCS1 \\
\hline rs1029871 & 52772674 & 212847 & .965 & 1 & .375 & Nonsynonymous & NEK4 \\
\hline
\end{tabular}

LD, linkage disequilibrium; MAF, minor allele frequency; SNP, single nucleotide polymorphism; UTR, untranslated region.

the $5^{\prime}$ untranslated region (UTR) area of genes, with potential functional roles (Table 2 ).

\section{Discussion}

This study adds to the evidence that the region 3p21.1 is a susceptibility locus for BP. In our sample the OR (.89) was closer to 1 than in the BP dataset reported by McMahon et al. (6) (estimated to be .85 , which was likely an overestimation of the true genetic effect size as a consequence of the "winner's curse") $(29,30)$. Our study replicated the original findings in a new sample with a $p$ value of .003 , and the $p$ value of the combined sample-now reaching 6775 cases and 33,606 control subjects-is estimated to be $2.68 \times 10^{-9}$. Hence, in addition to ANK3, CACNA1C, NCAN, and ODZ4 $(3,4,9)$, the 3 p21. 1 is a locus with strong evidence for association with BP.

The SNP we genotyped, rs2251219, lay within PBRM1, a gene that is important for chromatin remodeling. The polybromo 1 protein coordinates key features common to all remodeling complexes and has an important role in transcriptional regulation (31), and frequent mutations of the gene have been implicated in renal carcinoma (32). However, because rs2251219 is a synonymous SNP, it is highly likely that it acts as a proxy marker for one or more other genetic variants at the locus, which have functional role and a direct effect on disease susceptibility, and which have not yet been characterized. These might involve genes other than PBRM1.

The 3p21.1 locus lies within a gene-dense, highly conserved region with very low levels of genetic variation (33). This contains an extensive area of high LD spanning 276 kilobase pair on chromo- some 3 (Figure 2), containing at least 10 protein coding genes and several small nucleolar RNAs. These include STAB1/FELE-1, a probable scavenger receptor; GNL3 (guanine nucleotide binding proteinlike 3), a probable P53 interactor that encodes a protein that seems to be important for cell proliferation in central nervous system stem cells (34); GLT8D1, a member of the glycosyltransferase family of enzymes involved in the biosynthesis of carbohydrates and polysaccharides; SPCS1 (signal peptidase complex subunit 1 homolog); ITIH1 and other members of a family of inter-á (globulin) inhibitors; as well as several genes encoding proteins of unknown function (NT5DC2, LOC440957) and noncoding small RNA genes (small nucleolar RNA, C/D box genes SNORD19, 19B, and 69). Of the 195 SNPs in high LD $\left(r^{2}>.8\right)$ with $r 2251219$, there were 4 nonsynonymous SNPs, 3 SNPs that occur at splice sites, and 2 SNPs in the $5^{\prime}$ UTR area of genes, with potential functional roles (Table 2). Two of the nonsynonymous SNPs are in exons of GNL3; one nonsynonymous SNP and one potentially affecting a splice site are located within GLT8D1, and the fourth nonsynonymous SNP is within NEK4 (never in mitosis gene a-related kinase 4). Of these genes, GNL3 is the best candidate, because it is involved in proliferation of central nervous system cells (34), but considerable further mapping and functional biology analysis will be needed to identify which gene is causally implicated in disease risk.

A large body of evidence from genetic epidemiological studies suggests that diagnostic boundaries of psychiatric disorders are unclear and the pattern of heritability is complex. It has been hypothesized that some genetic variants might increase risk across

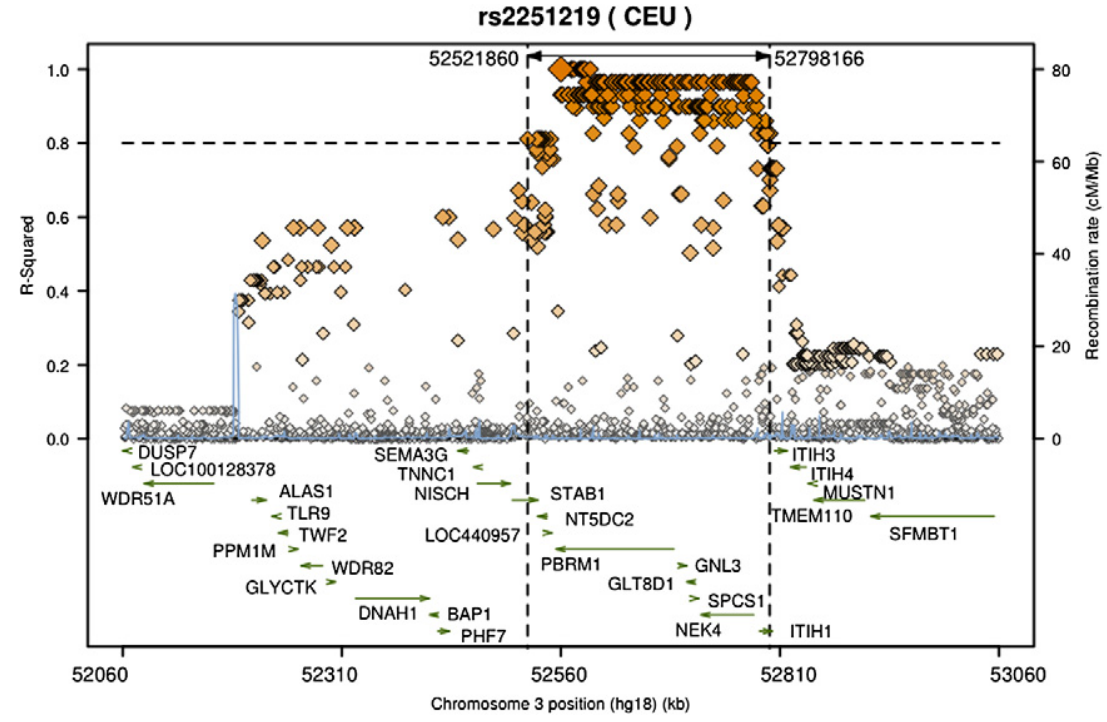

Figure 2. Plot of the genomic region showing an extensive area of high linkage disequilibrium containing at least 10 protein coding genes. 
several diagnoses, whereas others might be more specific to a subset of conditions $(35,36)$. Bipolar affective disorder has partial overlap in genetic risk with both schizophrenia (37) and major depression (38), and specific risk markers initially identified through GWAS of one disorder seem to be associated with a broader phenotype and have a cross-diagnosis effect. For example variation at CACNA1C influences susceptibility across the mood-psychosis spectrum (39).

In the recent meta-analysis by McMahon et al. (6) the authors suggested that rs2251219 is associated with major mood disorders including BP and major depression. However, by re-examining their data and analyzing additional samples with major depression, Breen et al. (7) argued that there is compelling evidence that 3p21.1 is a susceptibility locus for BP but not for major depression.

Similarly, Williams et al. (8) found association of this SNP with schizophrenia in a sample of 479 cases and 2937 control subjects with a stronger effect size $(\mathrm{OR}=.76)$. We examined the association with schizophrenia in a much larger dataset (8794 cases, 25,457 control subjects) but were unable to confirm this finding ( $O R=.97$, $p=.21$ ). Inclusion of the data by Williams et al. (8) gave nominally significant results (OR $=.96, p=.04)$, albeit with much smaller effect size than in BP (Supplementary material and Figure S1 in Supplement 1). Hence we conclude that there is strong evidence of association of rs 2251219 with BP but less compelling evidence of association with schizophrenia. This is in contrast to the results of two recent studies by the Psychiatric Genomics Consortium: genome-wide significant association was not found for either BP or schizophrenia, but association of SNPs in the 3p21.1 locus was genome-wide significant in combined BP and schizophrenia analyses $(9,40)$. The top SNPs flagged, rs736408 and rs2239547, are in LD with $\mathrm{rs} 2251219\left(r^{2}=.75\right.$ and .29 , respectively, in the European panel of the 1000 genomes project pilot dataset). The signal for rs736408 was strong in the bipolar sample (nominal $p=2 \times 10^{-7}$ ) but became genome-wide significant only after the addition of schizophrenia. Our view is that there is substantial evidence that the $3 p 21.1$ locus is associated with BP, but the association with a broader psychosis phenotype still needs further examination. A fuller understanding of the role of this locus in psychosis might require samples with more detailed phenotypes than dichotomous diagnostic information (41).

This work was partially funded by European Union Grant LSHM-CT2006-037761 (Project SGENE). EV was sponsored by a National Alliance for Research in Schizophrenia and Affective Disorders Young Investigator Award and the State Scholarship Foundation of Greece. The Romanian sample collection was funded by the Romanian Ministry for Education and Research (Grant number CNMP/UEFISCDI-42-151/2008, MG-S, CCD). Genotyping of the Dutch samples was sponsored by National Institute of Mental Health funding, R01 MH078075. The Dutch control subjects were derived from the Nijmegen Biomedical Study. Principal investigators of the Nijmegen Biomedical Study are L.A.L.M. Kiemeney, M. den Heijer, A.L.M. Verbeek, D.W. Swinkels, and B. Franke.

For the Norwegian sample we thank the study participants of the Thematically Organized Psychosis Study group and personnel involved in data collection and logistics, especially Thomas Doug Bjella and Eivind Bakken.

This work was supported by the Oslo University Hospital-Ullevål, Eastern Norway Health Authority (\#2004-123), the Research Council of Norway(\#167153/V50, 163070/V50, 183782/V50), and Eli Lilly for parts of the genotyping costs. Sample collection and genotyping of the Danish (Aarhus) schizophrenia sample of SGENE plus was sponsored by The Danish Strategic Research Council. Marc De Hert and Ruud van Winkel were supported by an unrestricted research grant from Astra-
Zeneca Belgium. SC, MMN, and MR were supported by the German Federal Ministry of Education and Research (BMBF), within the context of the National Genome Research Network 2 (NGFN-2), the National Genome Research Network plus (NGFNplus), and the Integrated Genome Research Network (IG) MooDS (Grant $01 G S 08144$ to SC and MMN, Grant $01 G S 08147$ to MR).

Genetic Risk and Outcome in Psychosis (GROUP) Consortium members: René S. Kahn, Don H. Linszen, Jim van Os, Durk Wiersma, Richard Bruggeman, Wiepke Cahn, Lieuwe de Haan, Lydia Krabbendam, and Inez Myin-Germeys.

All authors report no biomedical financial interests or potential conflict of interest.

\section{Supplementary material cited in this article is available online.}

1. Ioannidis JP, Thomas G, Daly MJ (2009): Validating, augmenting and refining genome-wide association signals. Nat Rev Genet 10:318-329.

2. WTCCC (2007): Genome-wide association study of 14,000 cases of seven common diseases and 3,000 shared controls. Nature 447:661-678.

3. Ferreira MA, O'Donovan MC, Meng YA, Jones IR, Ruderfer DM, Jones $L$, et al. (2008): Collaborative genome-wide association analysis supports a role for ANK3 and CACNA1C in bipolar disorder. Nat Genet 40:10561058.

4. Cichon S, Muhleisen TW, Degenhardt FA, Mattheisen M, Miro X, Strohmaier J, et al. (2011): Genome-wide association study identifies genetic variation in neurocan as a susceptibility factor for bipolar disorder. $\mathrm{Am} J$ Hum Genet 88:372-381.

5. Scott LJ, Muglia $P$, Kong $X Q$, Guan W, Flickinger $M$, Upmanyu $R$, et al. (2009): Genome-wide association and meta-analysis of bipolar disorder in individuals of European ancestry. Proc Natl Acad Sci U S A 106:75017506.

6. McMahon FJ, Akula N, Schulze TG, Muglia P, Tozzi F, Detera-Wadleigh SD, et al. (2010): Meta-analysis of genome-wide association data identifies a risk locus for major mood disorders on 3p21.1. Nat Genet 42:128131.

7. Breen G, Lewis CM, Vassos E, Pergadia ML, Blackwood DH, Boomsma DI, et al. (2011): Replication of association of 3p21.1 with susceptibility to bipolar disorder but not major depression. Nat Genet 43:3-5; author reply, 5 .

8. Williams HJ, Craddock N, Russo G, Hamshere ML, Moskvina V, Dwyer S, et al. (2011): Most genome-wide significant susceptibility loci for schizophrenia and bipolar disorder reported to date cross-traditional diagnostic boundaries. Hum Mol Genet 20:387-391.

9. Sklar P, Ripke S, Scott LJ, Andreassen OA, Cichon S, Craddock N, et al. (2011): Large-scale genome-wide association analysis of bipolar disorder identifies a new susceptibility locus near ODZ4. Nature Genet 43: 977-983.

10. Stefansson H, Ophoff RA, Steinberg S, Andreassen OA, Cichon S, Rujescu D, et al. (2009): Common variants conferring risk of schizophrenia. $\mathrm{Na}$ ture 460:744-747.

11. World Health Organization (1992): The ICD-10 Classification of Mental and Behavioural Disorders: Clinical Descriptions and Diagnostic Guidelines. Geneva: WHO.

12. American Psychiatric Association (2000): Diagnostic and Statistical Manual of Mental Disorders, 4th ed., Text Revision (DSM-IV). Washington, DC: APA.

13. World Health Organization. Division of Mental Health (1999): Schedules for Clinical Assessment in Neuropsychiatry (Version 2.1). Geneva: WHO Assessment, Classification and Epidemiology.

14. McGuffin P, Katz R, Aldrich J (1986): Past and present state examination: The assessment of 'lifetime ever' psychopathology. Psychol Med 16:461-465.

15. McGuffin P, Farmer A, Harvey I (1991): A polydiagnostic application of operational criteria in studies of psychotic illness. Development and reliability of the OPCRIT system. Arch Gen Psychiatry 48:764-770.

16. Spitzer RL, Endicott J, Robins E (1978): Research diagnostic criteria: rationale and reliability. Arch Gen Psychiatry 35:773-782.

17. Spitzer RL (1979): Schedule for Affective Disorders and Schizophrenia: LifeTime Version, 3rd ed. New York: State Psychiatric Institute.

18. Peters L, Andrews G (1995): Procedural validity of the computerized version of the Composite International Diagnostic Interview (CIDI-Auto) in the anxiety disorders. Psychol Med 25:1269-1280. 
19. World Health Organization (1993): Composite International Diagnostic Interview (CIDI): Interviewer's Manual. Geneva: WHO.

20. First MB, Spitzer, Robert L, Gibbon Miriam, and Williams, Janet B.W (2002): Structured Clinical Interview for DSM-IV-TR Axis I Disorders, Research Version, Patient Edition. (SCID-I/P). New York: Biometrics Research, New York State Psychiatric Institute.

21. Spitzer RL, Williams JB, Kroenke K, Linzer M, deGruy FV 3rd, Hahn SR, et al. (1994): Utility of a new procedure for diagnosing mental disorders in primary care. The PRIME-MD 1000 study. JAMA 272:1749-1756.

22. Djurovic S, Gustafsson O, Mattingsdal M, Athanasiu L, Bjella T, Tesli M, et al. (2010): A genome-wide association study of bipolar disorder in Norwegian individuals, followed by replication in Icelandic sample. J Affect Disord 126:312-316.

23. Grigoroiu-Serbanescu M, Herms S, Muhleisen TW, Georgi A, Diaconu CC, Strohmaier J, et al. (2009): Variation in P2RX7 candidate gene (rs2230912) is not associated with bipolar I disorder and unipolar major depression in four European samples. Am J Med Genet B Neuropsychiatr Genet 150B:1017-1021.

24. Gretarsdottir S, Thorleifsson G, Reynisdottir ST, Manolescu A, Jonsdottir S, Jonsdottir T, et al. (2003): The gene encoding phosphodiesterase 4D confers risk of ischemic stroke. Nat Genet 35:131-138.

25. Higgins JP, Thompson SG, Deeks JJ, Altman DG (2003): Measuring inconsistency in meta-analyses. BMJ 327:557-560.

26. StataCorp (2007): Stata Statistical Software: Release 10. College Station, Texas: StataCorp.

27. Steinberg $S$, de Jong $S$, Andreassen OA, Werge T, Borglum AD, Mors O, et al. (2011): Common variants at VRK2 and TCF4 conferring risk of schizophrenia. Hum Mol Genet 20:4076-4081.

28. Purcell S, Cherny SS, Sham PC (2003): Genetic Power Calculator: Design of linkage and association genetic mapping studies of complex traits. Bioinformatics 19:149-150.

29. Xiao R, Boehnke M (2009): Quantifying and correcting for the winner's curse in genetic association studies. Genet Epidemiol 33:453- 462.
30. Ioannidis JP, Ntzani EE, Trikalinos TA, Contopoulos-loannidis DG (2001): Replication validity of genetic association studies. Nat Genet 29:306309.

31. Thompson M (2009): Polybromo-1: The chromatin targeting subunit of the PBAF complex. Biochimie 91:309-319.

32. Varela I, Tarpey P, Raine K, Huang D, Ong CK, Stephens P, et al. (2011): Exome sequencing identifies frequent mutation of the SWI/SNF complex gene PBRM1 in renal carcinoma. Nature 469:539-542.

33. Durbin RM, Abecasis GR, Altshuler DL, Auton A, Brooks LD, Gibbs RA, et al. (2010): A map of human genome variation from population-scale sequencing. Nature 467:1061-1073.

34. Tsai RY, McKay RD (2002): A nucleolar mechanism controlling cell proliferation in stem cells and cancer cells. Genes Dev 16:2991-3003.

35. Burmeister M, McInnis MG, Zollner S (2008): Psychiatric genetics: Progress amid controversy. Nat Rev Genet 9:527-540.

36. Craddock N, Owen MJ (2010): The Kraepelinian dichotomy-going, going... but still not gone. Br J Psychiatry 196:92-95.

37. Lichtenstein P, Yip BH, Bjork C, Pawitan Y, Cannon TD, Sullivan PF, et al. (2009): Common genetic determinants of schizophrenia and bipolar disorder in Swedish families: A population-based study. Lancet 373: 234-239.

38. McGuffin P, Rijsdijk F, Andrew M, Sham P, Katz R, Cardno A (2003): The heritability of bipolar affective disorder and the genetic relationship to unipolar depression. Arch Gen Psychiatry 60:497-502.

39. Green EK, Grozeva D, Jones I, Jones L, Kirov G, Caesar S, et al. (2010): The bipolar disorder risk allele at CACNA1C also confers risk of recurrent major depression and of schizophrenia. Mol Psychiatry 15:1016-1022.

40. Ripke S, Sanders AR, Kendler KS, Levinson DF, Sklar P, Holmans PA, et al. (2011): Genome-wide association study identifies five new schizophrenia loci. Nature genetics 43:969-976.

41. Bilder RM, Sabb FW, Parker DS, Kalar D, Chu WW, Fox J, et al. (2009): Cognitive ontologies for neuropsychiatric phenomics research. Cogn Neuropsychiatry 14:419-450. 